\title{
Plasmonic Interactions: From Molecular Plasmonics and Fano Resonances to Ferroplasmons
}

\author{
Nicolás Passarelli, Luis A. Pérez, and Eduardo A. Coronado* \\ Córdoba 5000, Argentina

\begin{abstract}
Plasmon interactions are a subject of great interest from both the technological as well as the fundamental points of view. In this Perspective, we outline the great variety of physical phenomena that are produced by the interactions of localized surface plasmon resonance with molecular excitons; with other plasmonic nanostructures, particularly the Fano effect; and with nonplasmonic nanoparticles, such as the just-reported interaction with ferromagnetic nanoparticles. The theoretical as well as experimental challenges remaining to be elucidated are discussed.
\end{abstract}

INFIQC, Centro Laser de Ciencias Moleculares, Departamento de Fisicoquímica, Facultad de Ciencias Químicas, Universidad Nacional de Córdoba,

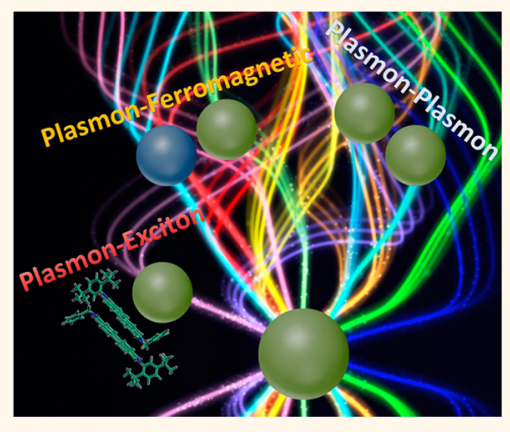

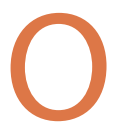
ne of the most remarkable nanoscale phenomenon with metals at optical wavelengths is the appearance of the so-called localized surface plasmon resonance (LSPR), which is related to the collective electronic excitation of the conduction electrons in response to an applied electromagnetic field, together with the highly confined and enhanced electromagnetic field that can be achieved by illuminating these nanostructures at suitable wavelengths. ${ }^{1}$ This phenomenon has given rise to a fast-developing field in nanooptics denoted as plasmonics. The extreme tunability of the LSPR for noble metals nanostructures, especially those made from silver and gold upon subtle changes in size, shape, and dielectric environment, make these plasmonic nanostructures suitable for a variety of applications including surface plasmon enhanced spectroscopies such as surface-enhanced Raman spectroscopy (SERS), tip-enhanced Raman spectroscopy (TERS), ${ }^{2}$ and metal-enhanced fluorescence (MEF). Triggered by these potential applications, a plethora of new phenomena have emerged from the interactions of these plasmonic nanostructures with different chemical entities or nanomaterials such as molecules (molecular plasmonics), ${ }^{3}$ photoactive molecules or semiconductors (excitons), ${ }^{4}$ other plasmonic nanoparticles (NPs), ${ }^{5,6}$ and nonplasmonic NPs (ferromagnetic or magnetic NPs). ${ }^{7}$ The extraordinary new phenomena characteristic of the surface plasmon interactions with the above-mentioned entities have made it possible to achieve ultrasensitive detection of molecules (even reaching the single-molecule detection limit), new plasmonic and tunable plasmonic filters, and plasmon waveguides, etc.).

In addition, a variety of electrodynamics and quantum methods to describe plasmon interactions, several bottom-up and topdown methods to fabricate plasmonic nanostructures of different architectures with nanoscale control, ${ }^{8}$ and more rigorous and precise techniques for characterizing their morphology and optical response have all emerged in the past decade. ${ }^{9}$ These developments are now not only able to give a one-to-one correlation between the optical response of a single-nanostructure entity and its morphology, but also to enable a direct comparison with theory and, in some cases, to give unexpected results that enable the development of more realistic

\section{* Address correspondence to coronado@fcq.unc.edu.ar.}

Published online October 17, 2014 10.1021/nn505145v

C일 2014 American Chemical Society 
theoretical models for the accurate physical interpretation of the optical measurements.

\section{The coupling of}

localized surface

plasmon resonance and molecular excitons is another important field where novel hybrid optical states can be developed.

A recent example that combines accurate morphological characterization with Rayleigh scattering and Raman microscopy at the single-NP level is the use of electron tomography to reconstruct the precise shape of a Au NP together with measurements using dark-field optical spectroscopy and Raman scattering of probe molecules adsorbed on the NP surface. ${ }^{10}$ This morphological reconstruction, together with a suitable electrodynamic model able to capture the details of the NP morphology as well as its interaction with the substrate, has been demonstrated to give quantitative correlation between theory and the experimental Rayleigh scattering response and the near-field enhancement of the Raman signal experienced around the NP surface. The use of electron energy loss spectroscopy (EELS) combined with scanning tunneling microscopy constitutes another powerful tool to investigate surface plasmons. This technique provides a map of the near electromagnetic field around a metal NP. Considerable progress has also been made recently to enhance the capabilities of TERS toward single-molecule detection. The largest benefit to single-molecule and single-particle spectroscopy is the ability to untangle properties that are averaged over simultaneously occurring events. Thus, single-molecule and single-particle spectroscopy enables observation of physical properties of small populations, which are otherwise obscured in ensemble experiments. For instance, singlemolecule detection is important in the field of plasmonics to characterize the hot spot and understand the ultrahigh enhancements observed in surface-enhanced techniques. Single-molecule studies can also contribute to our understanding of chemical catalysts to enable engineering of highly efficient novel catalysts, which are important to materials science and sustainability. Single-particle experiments enable quantitative examinations of the relationship between NP structure and optical properties and can yield predictive design rules. Additionally, in biology, eliminating ensemble averaging enables further insight into cellular processes such as enzyme catalysis and conformational studies.

The coupling of LSPR and molecular excitons is another important field where novel hybrid optical states can be developed. ${ }^{11,12}$ The nature of these modes is neither plasmonic nor excitonic, rather they act in a collective way as new eigenmodes. ${ }^{11}$ These hybrid nanomaterials have the ability to localize light with coherence features that can be narrower than pure plasmonic systems because of the presence of excitons that have bandwidths an order of magnitude narrower than those of LSPR alone. $^{12}$ The ultrafast response of the plasmon and excitons (on the order of several femtoseconds) facilates modulation of this new hybrid state on this very fast time scale. ${ }^{13}$ The combination of these ultrafast processes with the highly confined near field in this hybrid material makes possible a remarkable alteration of the energy redistribution process as well as efficient control of the exciton lifetime. A variety of applications in this area are in progress, such as achieving plasmon control of photochemical reactions, sensing, and optical gain.
The most well-known examples of plasmon interactions are the interactions of two nanostructures of the same metal, a phenomenon that gives rise to new hybridized plasmon modes. ${ }^{14}$ Perhaps one of the most interesting phenomena is the Fano effect, which can be produced from the interference between super-radiant modes and subradiant modes or from the interaction of dark and bright modes. ${ }^{15}$ Fano resonances have also been reported in multishell nanostructures, in cavities, as well as in heterodimers of noble metals. ${ }^{6,16}$ One of the first theoretical predictions of Fano resonances produced by localized surface plasmon coupling is in dolmen-type slab arrangements and in the nonconcentric ring/disk cavity system. ${ }^{17}$ One interesting type of nanostructure that can display sharp Fano resonances is clusters of plasmonic NPs (oligomers). These oligomers exhibit surface plasmon modes that are linear combinations of each constituent NP, provided that the interparticle separation is sufficiently small. Group theory is an important tool in analyzing the optical response of symmetric oligomers by classifying each linear combination of modes. A representative example of this is the symmetric heptamer formed by a central NP surrounded by six identical satellite nanoparticles. ${ }^{18}$ Symmetry arguments enable us to deduce that the hybridized collective plasmon mode is the result of the interaction of the central NP plasmon with the collective plasmons of the six-particle ring structure. Two cases can be analyzed here: the bonding mode and the antibonding mode. In the bonding mode, the dipole mode of the central NP is almost equal to the ring collective mode, giving rise to individual dipole NP plasmons all oscillating in phase along the polarization direction of the incident field (Figure 1a). This mode is superradiant and thus exhibits a strong radiative damping and is broadened into a continuum. In the antibonding 

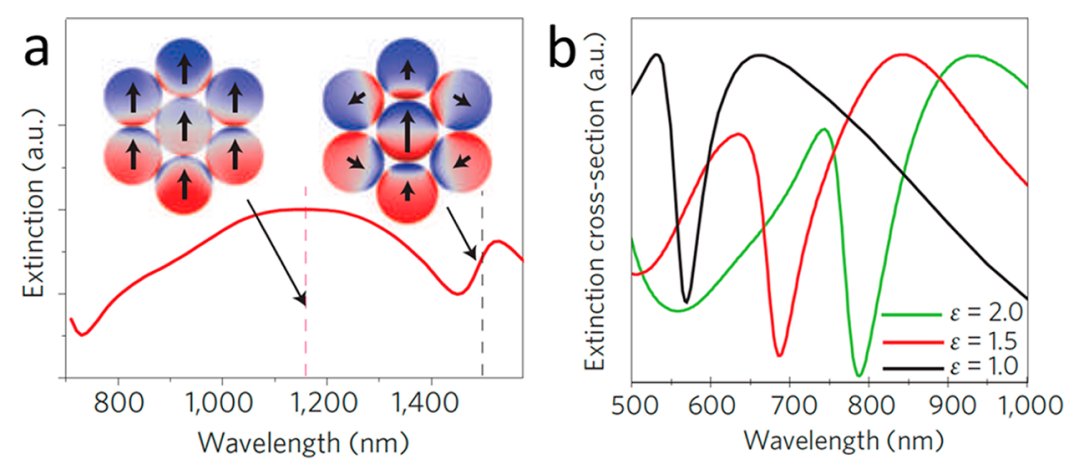

Figure 1. (a) Calculated dipole amplitudes of the bonding and antibonding collective dipolar plasmon modes in a gold nanoshell heptamer. (b) Effect of a surrounding dielectric medium on the extinction spectrum of a silver nanosphere heptamer. Reprinted with permission from ref 18. Copyright 2010 Nature Publishing Group.

mode, the dipolar plasmon of the central NP oscillates out of phase with respect to the dipolar mode of the surrounding NPs in the ring. Therefore, the net dipole moment of this mode is almost zero and results in a subradiant plasmon mode that induces a sharp Fano resonance when coupled to the almost continuum of the super-radiant (bonding) mode. $^{18}$

One practical way to measure the sensitivity of plasmon resonances to changes in the refractive index is the figure of merit (FOM), defined by the plasmon shift induced per unit change in the refractive index. The potential of the Fano resonance to be used as a plasmonic sensor is evidenced by the large and clearly discernible shifts observed by changing the surrounding media, where a high FOM can be obtained (Figure 1b). Fano resonances arising from the interference between a nonradiative mode and a continuum of radiative electromagnetic waves are also able to confine light more efficiently and are characterized by steeper dispersion than conventional plasmon resonances, which makes them promising for nanoscale biochemical sensing, ${ }^{19}$ switching and lasing applications.

In this issue of ACS Nano, Sachan et al. ${ }^{7}$ report an intriguing new phenomenon emerging as a result of the interaction of a ferromagnetic NP with a noble metal nanostructure: the appearance of a ferroplasmon, a strong and localized surface plasmon resonance in the visible range. The reasons, given by the authors, for a new name are (1) these plasmons appear at energies within the visible range, with large scattering intensities and extremely narrow bandwidths not present in isolated Co or CoFe alloy NPs, and (2) this phenomenon seems to be different from magnetoplasmonic effects in ferromagnets that emerge as a consequence of Kerr rotation.

The work by Sachan et al. demonstrates that a difference with respect to the new resonances that are produced by hybridization of two (sharp) plasmon modes in heterodimers formed by two nanoparticles of a different noble metal is that sharp resonance modes can be excited in materials with nonexistent/highly damped surface plasmon modes when this nonplasmonic NP is in contact with another NP having a sharp plasmon resonance.

The authors used EELS in a scanning transmission electron microscope to give precise nanoscale information on the local plasmonic behavior of the bimetallic nanostructures.

From the experimental EELS (Figure 2a,c) at the marked locations indicated in the HAADF image shown in Figure 2b, it can be seen that a peak at $2.7 \mathrm{eV}$ appears in the Co side of the bimetallic NP. This peak is absent in the EELS spectra of pure Co NP. The far-field optical spectrum of an array of these bimetallic NPs (Figure 2d) also depicts a resonance at an energy close to that of the EELS spectra $(2.57 \mathrm{eV})$
In this issue of $A C S$

Nano, Sachan et al.

report an intriguing

new phenomenon

emerging as a result of

the interaction of a

ferromagnetic

nanoparticle with a

noble metal

nanostructure: the

appearance of a

ferroplasmon, a strong

and localized surface

plasmon resonance in

the visible range.

(considering that this latter value is an ensemble average energy from particles with a distribution of diameters, while the former value is from individual nanoparticles of a fixed diameter). Quantification of the scattering intensities of the various plasmon peaks observed in the 2-4 eV range for the ferroplasmonic nanostructure consisting of a FeCo-Ag composite demonstrates two important features. First, the scattering intensity of the ferroplasmon mode at $2.6 \mathrm{eV}$ is most important at the particle-vacuum interface rather than in the interior side of the whole nanostructure. 


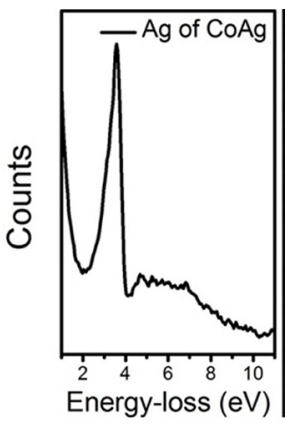

(a)

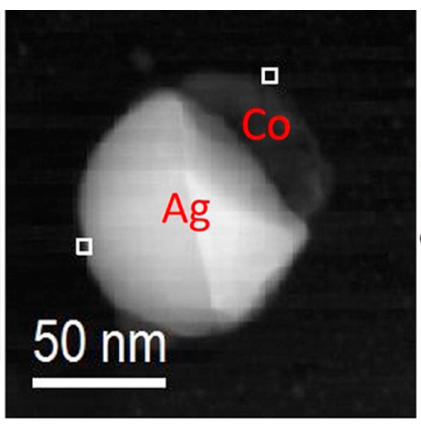

(b)

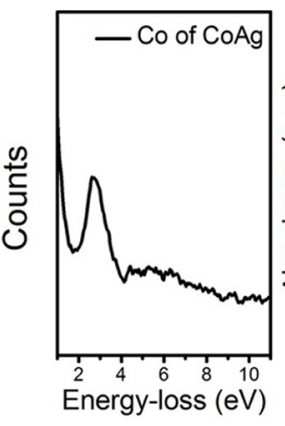

(c)

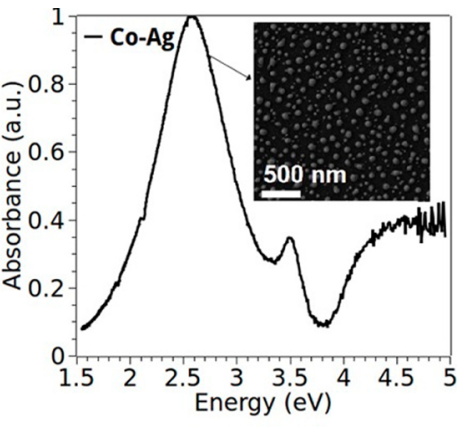

(d)

Figure 2. (a) Experimental EELS spectra from the marked location on the surface of the Ag region of the Co-Ag NP shown in (b). (b) HAADF image of an isolated Co-Ag NP on C-substrate. The marked regions correspond to the locations of the EELS spectra shown in (a) or (c). (c) Experimental EELS spectra from the marked location on the surface of the Co region of the Co-Ag NP shown in (b). (d) Far-field optical spectra for an ensemble of Co-Ag NPs on C-substrate. An SEM image of the Co-Ag NP array is shown in the inset. Reprinted from ref 7. Copyright 2014 American Chemical Society.
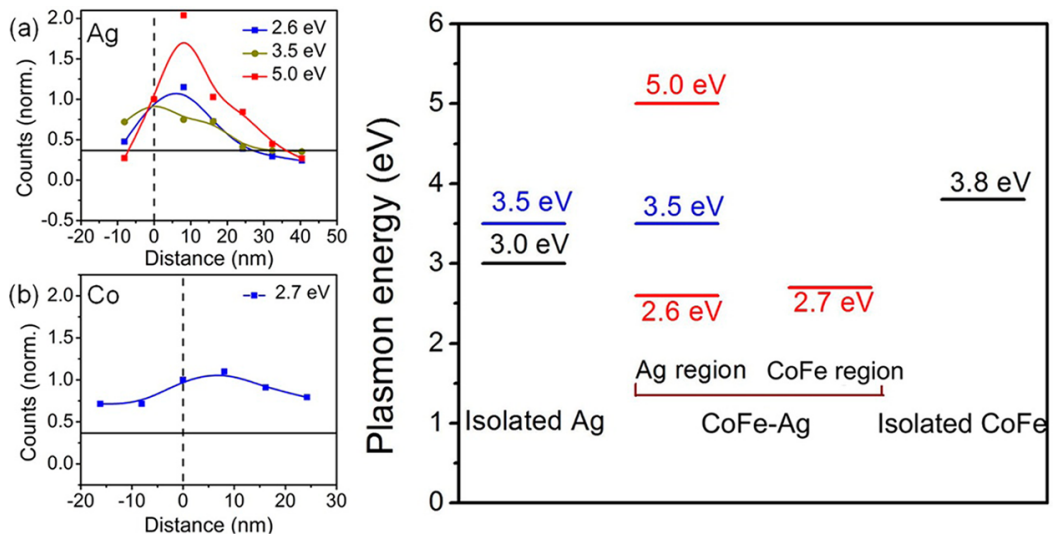

Figure 3. ( $a$ and $b$ ) Spatial dependence of the normalized intensities (w.r.t. the intensity at the surface) of various energy loss peaks within and outside of a CoFe- Ag NP (the vertical dashed line is the particle/vacuum interface and the horizontal solid line defines where the intensity has dropped to 1/e). (a) Surface and bulk plasmon decay trend from the Ag surface/vacuum interface and (b) surface and bulk plasmon decay trend from the Co surface/vacuum interface. The decay intensity measurements were made in the same way as those shown in Figure 1b, going from bulk of CoFe-Ag NP to vacuum. (c) Surface plasmon energy diagram showing the measured energy levels in $\mathrm{Ag}$ and CoFe regions of bimetallic $\mathrm{CoFe}-\mathrm{Ag}$, isolated Ag, and isolated CoFe NPs. Reprinted from ref 7. Copyright 2014 American Chemical Society.

Second, the overall scattering intensity for the ferroplasmon mode in the CoFe region is almost equivalent to that observed in the $\mathrm{Ag}$ region. One important issue that can be elucidated by using EELS is distinguishing between bulk plasmons and surface plasmons modes by quantifying the intensity of the EELS peaks as a function of the distance within and outside the NP due to the high degree of localization of the plasmon excitation that can be achieved with this technique.

Excitation of localized surface plasmons produces a decay of the EELS signal that extends beyond the nanostructure surface while bulk plasmon modes are confined to the interior of the nanostructure and decay very quickly outside. This feature allowed Sachan et al. to conclude that the modes excited from both the silver side at 2.6, 3.5, and $5 \mathrm{eV}$, as well as the mode from the CoFe side, are localized surface plasmons (Figure 3a,b). In this way, it is possible to build a surface plasmon energy diagram for the isolated $\mathrm{Ag}$, in the $\mathrm{Ag}-\mathrm{CoFe}$ and for the isolated CoFe NPs, as depicted in Figure 3. This diagram shows that the plasmons excited in the isolated CoFe regions or $\mathrm{Ag}$ regions are quite different from those of the combined $\mathrm{Ag}-\mathrm{CoFe}$ nanostructure. The localized surface plasmon corresponding to Ag NP that is isolated at $3.5 \mathrm{eV}$ does not change in the $\mathrm{Ag}$ region of the hybrid $\mathrm{Ag}-\mathrm{FeCo}$ nanostructure. The remarkable result is that this resonance does not appear in the CoFe isolated NP, but a strong localized surface plasmon resonance at $2.7 \mathrm{eV}$ is supported in the $\mathrm{CoFe}$ region of the $\mathrm{Ag}-\mathrm{CoFe}$ ferromagnetic-plasmon

composite.

The qualitative theoretical explanation of the physical origin of the ferroplasmon phenomena employs existing analytical theories for plasmon interactions such as the plasmon hybridization model, ${ }^{14}$ Fano interference approach, and the dipole-dipole interaction model (see ref 7 for details). In this respect, the analytical approach relies on the interaction of two spherical NPs and the use of a noncontacting 

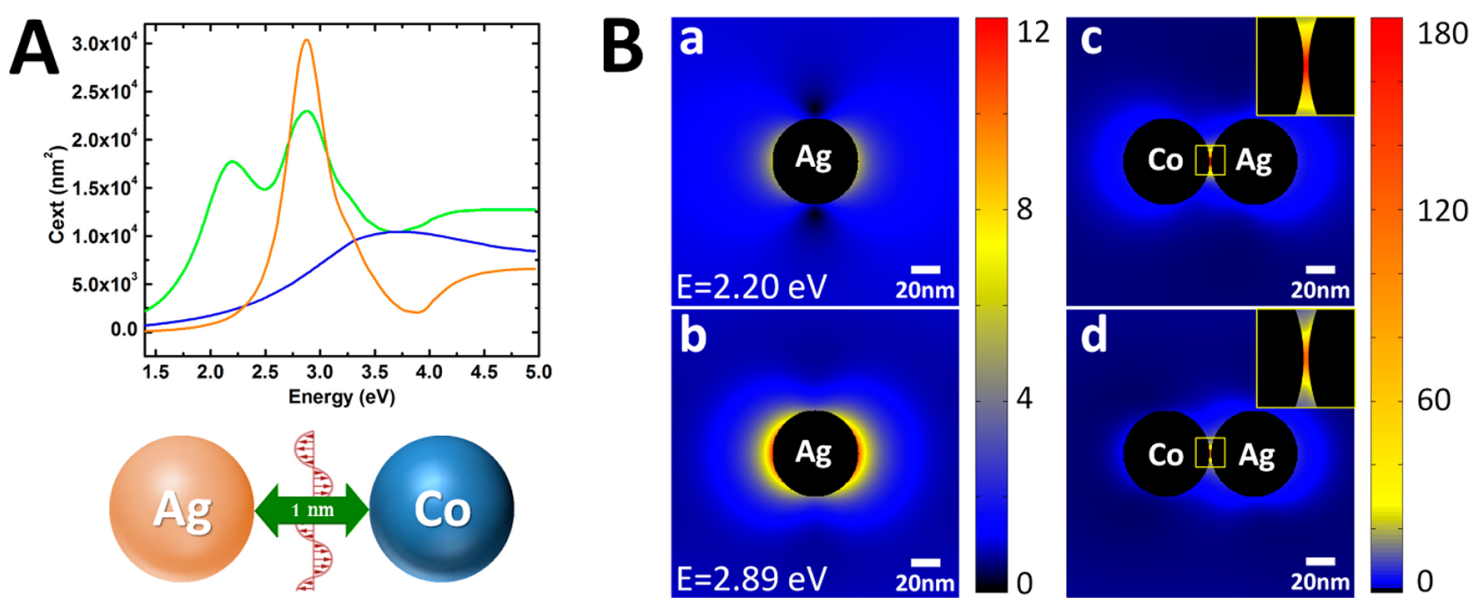

Figure 4. (A) Comparison between the extinction spectra of a single $60 \mathrm{~nm}$ diameter spherical silver nanoparticle (orange lines), a $60 \mathrm{~nm}$ spherical Co nanoparticle (blue line) and the extinction spectra of a dimer (green line) formed by the interaction of these two nanoparticles (NPs) separated by a $1 \mathrm{~nm}$ gap as illustrated in the bottom. The dimer depicts two peaks at 2.20 and $2.89 \mathrm{eV}$, the first one at a much lower energy than the peaks associated with the isolated Co NP and Ag NP and the second one at and energy also lower than the Co NP but at almost the same energy of the isolated Ag NP. (B) Electromagnetic field enhancement $\left(E / E_{0}\right)$ distribution around the nanoparticle dimer at the two peak energies produced by the interaction of a plasmonic NP and a nonplasmonic ferromagnetic Co NP shown in panel A. The insets in the figure show higher resolution views of the field enhancement around the nanoparticle dimer marked by the squares.

geometry, assuming that the contact between $\mathrm{Ag}$ and Co does not influence the interaction process. In this respect, the present experiments open the way for future implementation of more quantitative models for describing the interaction by taking into account the realistic, almost hemispherical shape of the NPs; considering the possibility of quantum mechanical tunneling at the interface between both metals (Co and $\mathrm{Ag}$ ); and including nonlocal effects in the dielectric constants. One possible route could be to use a combination of finite element methods such as the discrete dipole approximation or finite element time numerical approaches with recent approaches to include nonlocal effects in the dielectric response, ${ }^{20,21}$ taking the exact threedimensional geometry extracted from state-of-the-art electron tomography techniques. ${ }^{9}$

We have performed electrodynamic simulations that show that the interaction of a spherical Ag NP and a Co ferromagnetic nonplasmonic NP separated by a nanometer interparticle gap also gives rise to new plasmon modes. Figure 4 shows exact electrodynamics calculations using the generalized multiparticle Mie theory ${ }^{6,16}$ for the significant change of the extinction spectra produced by the interaction of a Ag NP with a Co NP, in a medium with a refractive index of 1.33 , both having the same diameter $(60 \mathrm{~nm})$. Qualitatively, the results are similar to those produced when the NPs are in contact (see ref 7). The hybridization of the Ag plasmonic mode at $2.89 \mathrm{eV}$ with the strongly damped plasmon mode of Co located at $3.74 \mathrm{eV}$ generates a lower energy LSPR mode at $2.2 \mathrm{eV}$ as the nanospheres approach each other at close distances, and another one at $2.89 \mathrm{eV}$, with an energy almost identical to the isolated Ag plasmon mode (Figure 4A). Remarkably, the near-field enhancement (defined as the ratio between the field in the vicinity of the NP, $E$, and the incident electromagnetic field, $E_{0}$ ) generated in the gap between the two spheres at each of the peak energies is almost 33 times greater than the enhancement produced in the isolated Ag spheres excited at the lower $(2.2 \mathrm{eV}$ ) energy modes or 10 times greater than the enhancement at the higher (2.89 eV) energy modes, respectively (Figure 4B). This feature demonstrates that if a plasmonic NP and a ferromagnetic NP are close enough, it is possible to obtain a considerable degree of enhancement and confinement of the near field without the necessity for any physical contact. Therefore, it should be possible to use this NP aggregate as a SERS substrate with significant enhancement and, at the same time, to give it additional functionality due to the ferromagnetic character of the Co NP.

From the technological point of view, one could envision several potential applications employing ferroplasmonic materials. One of them is in the field of magnetic data storage. Current hard disk devices (HDDs) with capacities of several terabits of information have data storage cells with dimensions of around $100 \mathrm{~nm} \times 100 \mathrm{~nm}$. Reducing the grain sizes of the magnetic materials to reduce the bit cell could increase the capacities of current HDDs; a problem, however, is that storage for materials with grains below a certain size becomes unstable and their magnetization can change arbitrarily due to thermal effects. ${ }^{22}$ With the application of a combination of plasmon-induced heating by light with ferromagnetic Co nanoislands, the limitations imposed by the grain size can be overcome as recently demonstrated by Stype et al., who developed a magnetic recording device at $1.5 \mathrm{~Pb} \mathrm{~m}^{-2}$ 
using an integrated plasmonic antenna. $^{23}$ The present discovery of ferroplasmons outlined in this issue of ACS Nano should certainly inspire future work in this direction.

Conflict of Interest: The authors declare no competing financial interest.

Acknowledgment. Authors acknowledge financial support of CONICET (PIP 112-201101-00430), FONCYT (PICT 20122286), SECYT-UNC and PME 1544-2006. N.P. and L.A.P. acknowledges CONICET for being recipients of a Ph.D. fellowship.

\section{REFERENCES AND NOTES}

1. Kelly, K. L.; Coronado, E. A.; Zhao, L. L.; Schatz, G. C. The Optical Properties of Metal Nanoparticles: The Influence of Size, Shape, and Dielectric Environment. J. Phys. Chem. B 2003, 107, 668-677.

2. Sonntag, M. D.; Pozzi, E. A.; Jiang, N.; Hersam, M. C.; Van Duyne, R. P. Recent Advances in Tip-Enhanced Raman Spectroscopy. J. Chem. Phys. Lett. 2014, 5, 3125-3130.

3. Sonntag, M. D.; Klingsporn, J. M.; Zrimsek, A. B.; Sharma, B.; Ruvuna, L. K.; Van Duyne, R. P. Molecular Plasmonics for Nanoscale Spectroscopy. Chem. Soc. Rev. 2014, 43, 1230-1247.

4. Wiederrecht, G. P.; Wurtz, G. A.; Bouhelier, A. Ultrafast Hybrid Plasmonics. Chem. Phys. Lett. 2008, 461, 171-179.

5. Taylor, R.; Lee, T.; Scherman, O. A.; Esteban, R.; Aizpurua, J.; Huang, F. M.; Baumberg, J. J.; Mahajan, S. Precise Subnanometer Plasmonic Junctions for SERS within Gold Nanoparticle Assemblies Using Cucurbit[n]uril "Glue". ACS Nano 2011, 5, 3878-3887.

6. Encina, E. R.; Coronado, E. A. On the Far Field Optical Properties of $\mathrm{Ag}-\mathrm{Au}$ Nanosphere Pairs. J. Phys. Chem. C 2010, 114, 16278-16284.

7. Sachan, R.; Malasi, A.; Ge, J.; Yadavali, S.; Krishna, H.; Gangopadhyay, A.; Garcia, H.; Duscher, G.; Kalyanaraman, R. Ferroplasmons: Intense Localized Surface Plasmons in MetalFerromagnetic Nanoparticles. ACS Nano 2014, 10.1021/nn5031719.

8. Zhao, P.; Li, N.; Astruc, D. State of the Art in Gold Nanoparticle Synthesis. Coord. Chem. Rev. 2013, 257, 638665.

9. Batenburg, K. J.; Bals, S.; Sijbers, J.; Kübel, C.; Midgley, P. A.; Hernandez, J. C.; Kaiser, U.; Encina, E. R.; Coronado, E. A.; VanTendeloo, G. 3D Imaging of Nanomaterials by Discrete Tomography. Ultramicroscopy 2009, 109, 730-740.

10. Perassi, E. M.; Hrelescu, C.; Wisnet, A.; Döblinger, M.; Scheu, C.; Jäckel, F.; Coronado, E. A.; Feldmann, J. Quantitative Understanding of the Optical Properties of a Single, Complex Shaped Gold Nanoparticle from Experiment and Theory. ACS Nano 2014, 8, 4395-4402.

11. Wurtz, G. A.; Evans, P. R.; Hendren, W.; Atkinson, R.; Dickson, W.; Pollard, R. J.; Zayats, A. V. Molecular Plasmonics with Tunable Exciton-Plasmon Coupling Strength in J-Aggregate Hybridized Au Nanorod Assemblies. Nano Lett. 2007, 7, 1297-1303.

12. Fofang, N. T.; Park, T.-H.; Neumann, O.; Mirin, N. A.; Nordlander, P.; Halas, N. J. Plexcitonic Nanoparticles: Plasmon-Exciton Coupling in Nanoshell-J-Aggregate Complexes. Nano Lett. 2008, 8, 3481-3487.

13. Balci, S.; Kocabas, C.; Küçüköz, B.; Karatay, A.; Akhüseyin, E.; Yaglioglu, H. G.; Elmali, A. Probing Ultrafast Energy Transfer between Excitons and Plasmons in the Ultrastrong Coupling Regime. Appl. Phys. Lett. 2014, 105, 051105-051110.

14. Prodan, E.; Radloff, C.; Halas, N. J.; Nordlander, P. A Hybridization Model for the Plasmon Response of Complex Nanostructures. Science 2003, 302, 419-422.

15. Lovera, A.; Gallinet, B.; Nordlander, P.; Martin, O. J. F. Mechanisms of Fano Resonances in Coupled Plasmonic Systems. ACS Nano 2013, 7, 4527-4536.

16. Encina, E. R.; Coronado, E. A. Near Field Enhancement in Ag Au Nanospheres Heterodimers. J. Phys. Chem. C 2011, 115, 15908-15914.

17. Hao, F.; Sonnefraud, Y.; Van Dorpe, P.; Maier, S. A.; Halas, N. J.; Nordlander, P. Symmetry Breaking in Plasmonic Nanocavities: Subradiant LSPR Sensing and a Tunable Fano Resonance. Nano Lett. 2008, 8, 3983-3988.

18. Luk'yanchuk, B.; Zheludev, N. I.; Maier, S. A.; Halas, N. J.; Nordlander, P.; Giessen, H.; Chong, C. T. The Fano Resonance in Plasmonic Nanostructures and Metamaterials. Nat. Mater. 2010, 9, 707-715.

19. Zhang, Y.; Zhen, Y.-R.; Neumann, O.; Day, J. K.; Nordlander, P.; Halas, N. J. Coherent Anti-Stokes Raman Scattering with Single-Molecule Sensitivity Using a Plasmonic Fano Resonance. Nat. Commun. 2014, 5, 4424-4431.

20. Mortensen, N. A.; Raza, S.; Wubs, M.; Søndergaard, T.; Bozhevolnyi, S. I. A Generalized Non-Local Optical Response Theory for Plasmonic Nanostructures. Nat. Commun. 2014, 5, 3809-3816.

21. Savage, K. J.; Hawkeye, M. M.; Esteban, R.; Borisov, A. G.; Aizpurua, J.; Baumberg, J. J. Revealing the Quantum Regime in Tunnelling Plasmonics. Nature 2012, 491, 574-577.

22. O'Connor, D.; Zayats, A. V. The Third Plasmonic Revolution. Nat. Nanotechnol. 2010, 5, 482-483.

23. Stipe, B. C.; Strand, T. C.; Poon, C. C.; Balamane, $\mathrm{H}_{\text {.; }}$ Boone, T. D.; Katine, J. A.; Li, J.-L.; Rawat, V.; Nemoto, H.; Hirotsune, A.; et al. Magnetic Recording at $1.5 \mathrm{~Pb} \mathrm{m^{-2 }}$ Using an Integrated Plasmonic Antenna. Nat. Photonics 2010, 4, 484-488. 